\title{
The current status of orphan drug development in Europe and the US
}

\author{
Anthony K Hall ${ }^{1, *}$, Marilyn R Carlson ${ }^{2}$ \\ ${ }^{1}$ PSR Orphan Experts, Hoofddorp, Netherlands and Findacure Development, Cambridge, UK; \\ ${ }^{2}$ Agility Clinical, Carlsbad, CA, USA.
}

\begin{abstract}
Summary Orphan drug legislation has been introduced in a number of countries in order to stimulate the development of treatments for rare diseases by introducing commercial incentives for companies wishing to undertake that development. In order to navigate the maze of regulatory regulations and procedures so that companies can make proper use of the orphan drug incentives, specialist knowledge is required. This article will review the current status of orphan drug development in the EU and the US, explain the incentives and procedures, and touch on the role of patient organisations in the process.
\end{abstract}

Keywords: Rare diseases, orphan drugs, orphan designation, patient organisations

\section{Introduction}

"Patients suffering from rare conditions should be entitled to the same quality of treatment as other patients". This laudable sentiment is enshrined in European law in the landmark European regulation $141 / 2000$ (1) which introduced a set of commercial incentives in European Union (EU) to try to stimulate the development of products for rare (orphan) diseases, which until then had been largely neglected by the pharmaceutical industry.

In the US, there are more than 25 million people affected by more than 7,000 diseases that are considered rare. With only 10 new drugs approved for rare diseases between 1972 and 1983, parents and caregivers enlisted the support of legislators and the Orphan Drug Act (ODA) became law in 1983 (2). The US was the first nation to introduce orphan drug legislation and, in the intervening years, a number of other countries have followed suit, for example Japan (1993), Singapore (1997), Australia (1998) and the EU (2000). Other countries, such as Canada, recognize the importance of

\footnotetext{
*Address correspondence to:

Dr. Anthony Hall, Co-founder, PSR Orphan Experts, Planetenweg 5, 2132HN Hoofddorp, Netherlands.

E-mail: tony.hall@psr-group.com

Co-founder, Findacure Development, 66 Devonshire Road,

Cambridge CB1 2BL, UK.

E-mail: tony@findacure.org.uk
}

orphan drug legislation and consider applications for rare diseases on a case-by-case basis, but have yet to issue regulations.

Whilst there is no doubt that the incentives have been of great benefit, the cause of the recent explosion of interest in orphan drug development is likely to be multifactorial, e.g. patent expiration for blockbuster drugs; the lack of innovative treatments for wellestablished diseases; the growth of the biotechnology industry; the advances in molecular biology; the considerable growth in the effectiveness of rare disease patient groups; and the ubiquitous availability of social media.

\section{Incentives for orphan drug development}

The main incentives of the orphan drug legislation in the EU and the US are shown in Table 1. The market exclusivity is usually considered to be the most important of the incentives, although this only comes into effect after marketing authorisation (MA) has been granted in the EU and after the new drug application (NDA) has been approved by the Food and Drug Administration (FDA) in the US.

In the EU, the market exclusivity prevents another application for a MA (and also the extension of an existing MA) for the same therapeutic indication, for a similar medicinal product. It is possible (and indeed quite common) for multiple products to obtain orphan designation for the same indication but, for two similar 
products, only the first to obtain MA will be granted the MA and the 10-year exclusivity. For example, in the EU, 16 products have received orphan designation for pulmonary arterial hypertension, but only 4 have received MA. A second product may be granted a MA for the same indication, if it is not similar to the first product, although it would still be necessary to demonstrate significant benefit if it was to obtain orphan designation. Exceptions to these rules exist if the original MA holder gives consent, or the original MA holder cannot supply sufficient product or the second applicant can show that their product is safer, more effective or otherwise clinically superior (5).

In the US, orphan drug designation is conferred to the active moiety in the product and, as in the EU, the first active moiety with orphan designation to reach the market receives the benefits of exclusivity. If a product has received marketing approval in the US for use in an orphan indication, the only way another product can be designated as an orphan drug for that indication is if the sponsor provides a reasonable hypothesis that their product is "clinically superior" to the approved product by means of greater effectiveness, greater safety, or that it provides a major contribution to patient care. Any claim for clinical superiority could require a head-tohead trial (6).

During the development programme, the free advice and fee reductions from the regulatory agencies can also be extremely helpful incentives. In the EU, these

Table 1. Key incentives of the orphan drug legislation in Europe and the US*

\begin{tabular}{lll}
\hline Items & EU & US \\
\hline Market exclusivity & 10 years ${ }^{\mathrm{a}, \mathrm{b}}$ & 7 years \\
Protocol assistance and follow-up & Yes & Yes \\
Reduced / waived regulatory fees & Yes & Yes \\
Tax credit on clinical trials & $\mathrm{No}$ & Yes \\
Specific subsidies for clinical trials & $\mathrm{No}^{\mathrm{c}}$ & Yes \\
\hline
\end{tabular}

${ }^{a}$ plus an extra 2 years if paediatric development included; ${ }^{b}$ may be reduced to 6 years if the product is sufficiently profitable; ${ }^{\mathrm{c}} \mathrm{EU}$ funding is available for rare diseases in addition to various national grants; ${ }^{\mathrm{d}}$ plus an additional 6 months of paediatric exclusivity for qualified studies. *Adapted from reference (3) and (4). incentives generally favour small and medium-sized enterprises (SMEs), but are revised from time to time as shown in Table 2.

In the US, orphan drug designation gives sponsors an exemption from the fees required when filing a new drug or biologic application. For fiscal year 2014, the fee for an application requiring clinical data is $\$ 2,169,100$ under the Prescription Drug User Fee Act (PDUFA).

The ODA is widely recognised as having been successful in encouraging the development of products for orphan indications in the US and the EU. Since it was enacted in 1983, there have been nearly 3,000 orphan designations and 448 approvals in the US. In 2008, more than a third of all FDA-approved new chemical entities (NCEs) were orphan drugs. In the EU, there have been 1,219 products designated and 78 approved (excluding those withdrawn and expired, there are currently 986 designated and 67 approved orphan drugs) (7).

\section{Obtaining orphan designation}

The incentives outlined above apply to products which have obtained designation as an orphan drug (orphan medicinal product in the EU). An orphan designation application may be submitted at any time in development, but before the submission of the application for marketing authorisation. In the US, the application for orphan designation is assessed by the Office of Orphan Products Development (OOPD), a branch of the FDA; in the EU it is assessed by the Committee for Orphan Medicinal Products (COMP), one of the committees of the EMA.

For a drug to qualify for orphan designation in the US, both the drug and the disease or condition must meet criteria specified in the US Code of Federal Regulations (CFR) Title 21 Part 316 Orphan Drugs (2). The regulations define "rare disease or condition" as any disease or condition which affects less than 200,000 individuals in the US, or if it affects more than 200,000 persons in the US, there is "no reasonable expectation"

Table 2. Recent changes in fee reductions in Europe for companies developing orphan medicinal products

\begin{tabular}{|c|c|c|}
\hline Items & 2013 & 2014 \\
\hline Protocol assistance and follow-up & $\begin{array}{l}100 \text { for SMEs } \\
40 \% \text { for non-SMEs } \\
\text { (non-paediatric-related assistance) } \\
100 \% \text { for non-SMEs } \\
\text { (paediatric-related assistance) }\end{array}$ & $\begin{array}{l}100 \text { for SMEs } \\
75 \% \text { for non-SMEs } \\
\text { (non-paediatric-related assistance) } \\
100 \% \text { for non-SMEs } \\
\text { (paediatric-related assistance) }\end{array}$ \\
\hline Pre-authorisation inspections & $100 \%$ for SMEs & $100 \%$ \\
\hline Initial marketing authorisation application & $100 \%$ for SMEs & $\begin{array}{l}100 \% \text { for SMEs } \\
10 \% \text { for non-SMEs }\end{array}$ \\
\hline $\begin{array}{l}\text { Post-authorisation activities, including } \\
\text { annual fee in the 1st year after MA }\end{array}$ & $100 \%$ for SMEs & $100 \%$ for SMEs \\
\hline
\end{tabular}


that the cost of developing and making the drug available in the US for this rare disease or condition will be recovered from US sales of the drug.

The prevalence of the disease for which the treatment is being developed must be less than 200,000 persons in the US who have been diagnosed at the time of the submission of the request for orphan drug designation. For a vaccine or a drug to prevent a rare disease or condition, the estimated number of people to whom the drug will be administered annually must be included with a basis for the estimate. When there is no reasonable expectation that the cost of research and development can be recovered by sales of the product in the US, the sponsor must submit detailed documentation of the development costs incurred and the anticipated market for the drug.

In the EU, the prevalence must be below 5 per 10,000 of the EU population, except (in a comparable way to the US law) where the expected return on investment is insufficient to justify the investment.

There has been a certain degree of harmonisation of the procedures for designation on either side of the Atlantic (e.g. the introduction of a common application form) but there remain some differences between the procedures, as outlined in Table 3.

One of the key differences between the orphan designation process in the EU and US is that, in the latter the emphasis is on demonstrating the scientific rationale and disease prevalence, whilst in the EU there are two additional requirements: $i$ ) that the condition is life-threatening or seriously debilitating, and $i i)$ that there is currently either no satisfactory method (of diagnosis, prevention or treatment) or that the new product will be of significant benefit over the existing method. Any product with a MA in any EU country would be considered to be a "satisfactory" method and, even where a product is not authorised, if it is widely used it may be considered to be satisfactory. In such a case, the applicant needs to provide a solid argument as to why the new method is expected to be superior.

The most challenging part of the application is usually obtaining reliable prevalence data. For the $\mathrm{EU}$, the criteria require demonstrating prevalence in the European Community and it is not enough to cite prevalence figures for one or two countries only. For many rare diseases, there may be very little information available in the literature on the epidemiology of the disease. It is not considered adequate to state that the prevalence "obviously" meets the criterion, nor to simply quote sources such as OrphaNet. Instead, it is necessary to provide a properly referenced analysis and, if the prevalence figure is close to the cut-off of 5 per 10,000 , some sensitivity analyses may also be needed to convince the COMP that the true prevalence is really within the limits.

The process of orphan designation can take up to six months in the EU. The sponsor needs to submit a Letter of Intent at least two months before the intended submission date, which allows time for the COMP to appoint the coordinators at one of its regular monthly meetings. Subsequently, a pre-submission meeting can be very helpful to informally discuss the draft application and obtain feedback from the coordinators on likely weaknesses in the application, so that these can be addressed before submission. After submission, there is a process of validation, during which the content of the application is examined for completeness and for conformity to the guidelines. Only after this

Table 3. Key differences in the procedures for orphan designation in the EU and US*

\begin{tabular}{|c|c|c|}
\hline Items & EU & US \\
\hline Terminology & Orphan medicinal product designation & Orphan drug designation \\
\hline Application to & Committee for Orphan Medicinal Products & Office of Orphan Products Development \\
\hline Timetable & $\begin{array}{l}\text { Timetable for submission and assessment published } \\
\text { by EMA }\end{array}$ & Any time; no defined timetable \\
\hline Prevalence criteria & $\begin{array}{l}\text { Disease or condition affects }<5 \text { in } 10,000 \text { persons in } \\
\text { the EU }\end{array}$ & $\begin{array}{l}\text { Disease or condition affects }<200,000 \text { persons in } \\
\text { the US }\end{array}$ \\
\hline Dossier & Sections A-E according to ENTR/6283/00 & Nine parts according to $21 \mathrm{CFR} 316.20$ \\
\hline Key aspects of the application & $\begin{array}{l}\text { Medical plausibility } \\
\text { Prevalence } \\
\text { Justification of significant benefit or why other } \\
\text { methods are not satisfactory }\end{array}$ & $\begin{array}{l}\text { Scientific rationale } \\
\text { Prevalence }\end{array}$ \\
\hline Sponsor established in territory & Proof of establishment in EU & Not required \\
\hline Translations & $\begin{array}{l}\text { Translations of product name and proposed orphan } \\
\text { indication into all official languages of the EU plus } \\
\text { Icelandic and Norwegian }\end{array}$ & Not required \\
\hline
\end{tabular}

"Adapted from references of $(2,4,8-10)$. 
does the clock start. Sixty days after the clock start, the COMP considers the application at its meeting and the application can receive a positive opinion at this stage. Quite commonly however, the COMP has some questions, which are communicated to the applicant as a List of Issues. The sponsor then has the opportunity to address these in writing and in person at an oral explanation, which occurs 90 days after the clock start at the next COMP meeting. The COMP is required to issue an opinion at this point. A positive opinion is sent to the European Commission (EC) for ratification, which is supposed to occur after a further 30 days. When a negative opinion from the COMP is inevitable, the applicant is given the opportunity to withdraw the application at that stage, in order to avoid the publication of a negative opinion.

In the US, the process is less complicated. A request for orphan designation is submitted in writing to the FDA OOPD. The sponsor may request orphan designation of a previously unapproved drug, or for a new use of an already marketed drug. In addition, a sponsor of a drug that is otherwise the same drug as an already approved drug may seek and obtain orphan drug designation for the subsequent drug for the same rare disease or condition if it can present a plausible hypothesis that its drug may be clinically superior to the first drug. More than one sponsor may receive orphandrug designation for the same drug for the same rare disease or condition, but each sponsor seeking orphandrug designation must file a complete request for designation. The application must include prevalence data and a scientific rationale that establishes a medically plausible basis to expect the drug will be effective for the rare disease. Clinical trial data is preferred but, in the absence of data in humans, "compelling" pre-clinical data in a relevant animal model may be adequate to support the scientific rationale. Animal toxicology data that describes the safety of the drug is not acceptable support for the scientific rationale.

For a drug used to treat a rare disease or condition, the application requires documentation with authoritative references of the prevalence of the disease or condition. For products to be used for a condition of less than one year in duration, incidence may be used as an estimate of the target population. For a product to be used as a preventative (e.g. a vaccine), the application requires documentation of the number of people to whom the drug will be administered annually. If the basis for the application is that there is no reasonable expectation of recovering the costs of development, justification must be provided for production and marketing costs the sponsor has incurred and expects to incur during the first seven years after the drug is marketed in the US (2).

To apply for designation in the US, the sponsor can follow the content and format described in the regulations (21 CFR 316.20) and use the common application (Form FDA 3671). The common application form includes items that are specific to the EU, however, if a sponsor is going to apply for orphan drug designation in both the EU and US, the common application form may be useful (11).

Once received by the OOPD, the application is assigned a designation application number, entered into the OOPD database, and an acknowledgement letter is sent to the sponsor. A reviewer is assigned and the reviewer prepares a review which is sent to the OOPD Team Leader for a second level review and concurrence. The review is forwarded for a third level review by the OOPD Office Director. Following the OOPD Director's concurrence, a designation letter, a letter requesting additional information, or a denial letter is prepared for the Director's signature and the letter is issued to the sponsor. The information is not made public unless the orphan designation is granted. On the FDA website there is a searchable database listing all orphan designations and approvals (12).

Every foreign sponsor that plans to apply for orphan drug designation is required to have a US agent to correspond with the FDA on their behalf. The agent must be a permanent resident and may be an individual, a firm, or a domestic corporation and may represent any number of sponsors. The name of the permanentresident agent, address, telephone number, and email address must be submitted to the OOPD (13).

\section{After orphan designation}

Companies sometimes fix their objectives on obtaining orphan designation, as if this is the end of the process, when it is usually just the beginning. This may be because, for some small companies, it is an important milestone which can be used to attract investors. However, it is important to realise that the development of an orphan drug needs to be performed in the proper way to ultimately lead to a positive benefit-risk assessment by the regulatory agencies.

Some companies make the mistake of thinking that "orphan status" will automatically allow them to obtain an expedited MA/NDA based on a single small study. Although there are now many examples of orphan drugs which have been granted approval based on very small clinical development programmes, this is often due to the fact that the treatment effect can be quite large in these serious, often fatal diseases, for which there is no other therapeutic option. In the end, the regulators still need to be convinced that the product is safe and effective. This is one of the reasons why it is very useful to obtain protocol assistance (the equivalence of scientific advice for orphan drugs) from the European Medicines Agency (EMA) prior to embarking on a nonconventional development programme.

Similarly, in the US, the granting of an orphan designation request does not alter the standard regulatory requirements and process for obtaining 
marketing approval. Safety and effectiveness of a drug must be established through adequate and wellcontrolled studies (14).

\section{Special regulatory procedures}

Notwithstanding the above commentary, there are some special regulatory procedures which, although not specific for orphan drugs, are more likely to apply to products for rare diseases. Some of these procedures are common to both the EU and US, whilst others are specific for each territory, as shown in Table 4.

It is noteworthy that, of the 67 currently approved orphan medicinal products in the EU, 4 have conditional approval and 14 have MA under exceptional circumstances.

In 2012, FDA signed into law the Food and Drug Administration Safety and Innovation Act (FDASIA) which created a new expedited drug development tool, known as the "breakthrough therapy" designation (18). This new designation allows FDA to assist drug developers to expedite the development and review of new drugs that have preliminary clinical evidence that indicates the drug may offer a substantial improvement over available therapies for patients with serious or lifethreatening diseases.

In 2013, FDA issued a draft guidance that provides detailed information about breakthrough designation and other expedited approval programs; Expedited Programs for Serious Conditions — Drugs and Biologics June 2013 (19).

As shown in Table 5, the four programs intended to expedite development and review of drugs to address unmet need in the treatment of serious or life threatening conditions are fast track designation, breakthrough therapy designation, the accelerated approval pathway and priority review.

\section{National Plans for rare diseases}

Moving away from the purely regulatory aspects of orphan drugs, it is useful to have a look at the framework

Table 4. Comparison of regulatory procedures in the US and EU intended to accelerate the approval of drugs which fulfil an unmet medical need*

\begin{tabular}{|c|c|}
\hline FDA & EMA \\
\hline $\begin{array}{l}\text { Fast Track Designation } \\
\text { Rolling review to help expedite the process. } \\
\text { More meetings with FDA. }\end{array}$ & No equivalent \\
\hline $\begin{array}{l}\text { Breakthrough Therapy Designation } \\
\text { Intensive guidance on efficient drug development. } \\
\text { Organizational commitment involving senior managers. }\end{array}$ & No equivalent \\
\hline Accelerated Approval Pathway & Conditional Approval \\
\hline Approval with a surrogate or intermediate clinical endpoint. & Incomplete data; not specific for surrogate endpoint. \\
\hline $\begin{array}{l}\text { Approval is conditional on post-approval trials showing clinical } \\
\text { benefit, after which FDA grants a traditional approval. }\end{array}$ & $\begin{array}{l}\text { Approval is conditional on providing additional post-approval data. } \\
\text { After confirmation, authorisation is converted to a normal approval. }\end{array}$ \\
\hline No equivalent & $\begin{array}{l}\text { Approval under exceptional circumstances } \\
\text { Incomplete data. } \\
\text { It is not expected that compete data can ever be provided. }\end{array}$ \\
\hline Priority Review Designation & Accelerated Assessment \\
\hline Reduced time for review of NDA & Reduced time for review of MAA \\
\hline Reduced from 10 to 6 months & Reduced from 210 to 150 days \\
\hline
\end{tabular}

"Adapted from references of (15-17).

Table 5. FDA Expedited Programs for Serious Conditions

\begin{tabular}{|c|c|c|c|c|}
\hline Items & Fast track designation & Breakthrough therapy designation & Accelerated approval pathway & Priority review designation \\
\hline When to submit & $\begin{array}{l}\text { With IND and no later } \\
\text { than the pre-BLA or } \\
\text { pre-NDA meeting }\end{array}$ & $\begin{array}{l}\text { With IND and no later than the } \\
\text { end-of-Phase } 2 \text { meeting }\end{array}$ & $\begin{array}{l}\text { Discuss with FDA review } \\
\text { division }\end{array}$ & $\begin{array}{l}\text { With original BLA, NDA, } \\
\text { or efficacy supplement }\end{array}$ \\
\hline FDA response & $\begin{array}{l}\text { Within } 60 \text { calendar } \\
\text { days }\end{array}$ & Within 60 calendar days & Not specified & Within 60 calendar days \\
\hline Reference & $\begin{array}{l}\text { FDA Modernization } \\
\text { Act of } 1997 \text { (FDAMA), } \\
\text { amended by FDASIA }\end{array}$ & $\begin{array}{l}\text { Section } 506 \text { (a) of the FD\&C Act, } \\
\text { as added by FDASIA }\end{array}$ & $\begin{array}{l}21 \text { CFR part } 314 \text {, subpart } \mathrm{H} \text {; } \\
21 \mathrm{CFR} \text { part } 601 \text {, subpart E; } \\
\text { Section } 506(\mathrm{c}) \text { of the FD\&C } \\
\text { Act, as amended by FDASIA }\end{array}$ & $\begin{array}{l}\text { Prescription Drug User Fee } \\
\text { Act of } 1992\end{array}$ \\
\hline
\end{tabular}


within which the orphan drugs expansion is occurring. There is no doubt that the rare diseases patient groups have been instrumental in the creation, first of the orphan drugs legislation and later of the introduction and expansion of rare diseases services within Europe. EURORDIS and NORD, the umbrella organisations for rare diseases patient groups in Europe and the US, have been key players in lobbying for change.

One of the important consequences of the push for change was the publication of a European Commission recommendation that all EU countries should "elaborate and adopt a (national) plan or strategy as soon as possible, preferably by the end of 2013 at the latest, aimed at guiding and structuring relevant actions in the field of rare diseases within the framework of their health and social systems" (20). The recommendation also proposed the setting up of Centres of Expertise and European Reference Networks for rare diseases. The enthusiasm with which the recommendation has been taken up by individual Member States has been variable with, for example, France publishing its first National Plan for rare diseases in 2004, whilst the UK eventually published its first plan only in late 2013.

In addition to the umbrella groups, individual patient groups have also been very productive at a "grass roots" level, organising the setting up of National Centres, raising money to fund basic research and, in some cases, even driving the clinical development of treatments by establishing consortia of stakeholders which can together undertake large projects previously only possible by pharmaceutical companies (21).

\section{Pricing and value of orphan drugs}

It is inevitable that, when the total market for a new treatment is very small, as is the case for orphan drugs, the price that the manufacturer will charge is likely to be significantly higher than for drugs to treat common diseases. In many cases, the cost is several hundred thousand dollars/Euros per patient per year. With ever-increasing pressures on health budgets, the reimbursement bodies have started to look very critically at the cost-benefit ratio for orphan drugs, leading to a situation where many orphan drugs, although approved throughout Europe, are not available in some countries due to lack of funding.

In the US, patient access to expensive orphan drugs is limited by the patient's own lack of health insurance or their inability to pay the portion of the cost of their treatment not covered by their insurance plan.

Various approaches have been proposed to tackle the issue of "value" for orphan drugs, see for example Hughes-Wilson et al. (22), but to date none has been adopted. The prospect that, in the future, orphan drugs might find it increasingly difficult to get reimbursed is a potentially negative consequence of an otherwise very positive move to provide treatments for patients with rare diseases.

\section{Conclusion}

The orphan drug legislation in the US and the EU has been successful in enabling patients with rare diseases to receive treatments that would otherwise never have been developed. More than 30 years after the ODA was approved in the US and 14 years since the European Regulation, this has led to the approval of 448 orphan products in the US and 78 in Europe. In addition to the incentives within the original legislation, there are now additional regulatory pathways which help to expedite the development and approval of drugs for conditions of unmet medical need. In addition, the deeper involvement of patient organisations in the drug development process itself, rather than purely as lobbyists, is changing the way that orphan drugs are developed.

\section{References}

1. European Parliament and Council of the European Union. Regulation (EC) No 141/2000 of the European Parliament and of the Council of 16 December 1999 on orphan medicinal products. Official Journal of the European Communities. 2000; 22.1.2000.

2. US Code of Federal Regulations (CFR) Title 21 Food and Drugs Part 316 Orphan Drugs.

3. Center for Drug Evaluation and Research (CDER), Center for Biologics Evaluation and Research (CBER), Food and Drug Administration. Guidance for Industry: Qualifying for Pediatric Exclusivity Under Section 505A of the Federal Food, Drug, and Cosmetic Act. http:// www.fda.gov/downloads/Drugs/GuidanceComplianc eRegulatoryInformation/Guidances/UCM080558.pdf (accessed January 30, 2014).

4. Hall AK. The practicalities of clinical development of drugs for rare diseases. In: Rare Diseases: Challenges and Opportunities for Social Entrepreneurs (Sireau N, ed.). Greenleaf Publishing, Sheffield, UK, 2013; pp.62-86.

5. Commission of the European Communities (2000). Commission Regulation (EC) No 847/2000 of 27 April 2000 laying down the provisions for implementation of the criteria for designation of a medicinal product as an orphan medicinal product and definitions of the concepts "similar medicinal product" and "clinical superiority". Official Journal of the European Communities. 2000; 28.4.2000.

6. US Code of Federal Regulations (CFR) Title 21 Food and Drugs Part 316.31 Scope of Orphan Drug Exclusive Approval. http://www.accessdata.fda.gov/scripts/cdrh/ cfdocs/cfcfr/CFRSearch.cfm? fr $=316.31$ (accessed January 30, 2014).

7. EMA website http://www.ema.europa.eu/ema (accessed January 30, 2014).

8. European Commission Enterprise Directorate-General. Guideline on the Format and Content of Applications for Designation as Orphan Medicinal Products and on the Transfer of Designations from One Sponsor to Another (ENTR/6283/00 Rev 3; Brussels: European Commission, 
9 July 2007). http://ec.europa.eu/health/files/orphanmp/ doc/2007_07/format_content_orphan_applications_ rev3_200707_en.pdf(accessed January 30, 2014).

9. Committee for Orphan Medicinal Products (COMP). Recommendation on Elements Required to Support the Medical Plausibility and the Assumption of Significant Benefit for an Orphan Designation (EMA/ COMP/15893/2009 Final; London: European Medicines Agency, 2 March 2010). http://www.ema.europa.eu/docs/ en_GB/document_library/Regulatory_and_procedural_ guideline/2010/07/WC500095341.pdf (accessed January 30, 2014).

10. Committee for Orphan Medicinal Products (COMP). Points to Consider on the Calculation and Reporting of the Prevalence of a Condition for Orphan Designation (COMP/436/01; London: European Agency for the Evaluation of Medicinal Products, 26 March 2002). http://www.ema.europa.eu/docs/en_GB/document library/Regulatory_and_procedural_guideline/2009/09/ WC500003773.pdf (accessed January 30, 2014).

11. Common Orphan Application. http://www.fda.gov/ downloads/AboutFDA/ReportsManualsForms/Forms/ UCM048361.pdf (accessed January 30, 2014).

12. Searchable FDA Database for Orphan Designated and/ or Approved Products. http://www.accessdata.fda.gov/ scripts/opdlisting/oopd (accessed January 30, 2014).

13. US Code of Federal Regulations (CFR) Title 21 Food and Drugs Part 316.22 Permanent Resident Agent for Foreign Sponsor. http://www.accessdata.fda.gov/scripts/ cdrh/cfdocs/cfcfr/CFRSearch.cfm? fr $=316.22$ (accessed January 30, 2014).

14. Haffner ME. Adopting orphan drugs: Two dozen years of treating rare diseases. N Engl J Med. 2006; 354:445-447.

15. Commission of the European Communities. Commission Regulation (EC) No 507/2006 of 29 March 2006 on the conditional marketing authorisation for medicinal products for human use falling within the scope of Regulation (EC) No 726/2004 of the European Parliament and of the Council. Official Journal of the European Union. 2006; 30.3.2006.

16. Committee for Medicinal Products for Human Use (CHMP). Guideline on the Scientific Application and the Practical
Arrangements Necessary to Implement Commission Regulation (EC) No 507/2006 on the Conditional Marketing Authorisation for Medicinal Products for Human Use Falling Within the Scope of Regulation (EC) No 726/2004 (EMEA/509951/2006; London: CHMP, 5 December 2006). http://www.ema.europa.eu/docs/en_GB/document_library/ Scientific_guideline/2009/10/WC500004908.pdf (accessed January 30, 2014).

17. Committee for Medicinal Products for Human Use (CHMP). Guideline on Procedures for the Granting of a Marketing Authorisation Under Exceptional Circumstances, Pursuant to Article 14 (8) of Regulation (EC) No 726/2004 (EMEA/357981/2005; London: European Medicines Agency, 15 December 2005). http://www.ema.europa.eu/docs/en_GB/document library/Regulatory_and_procedural_guideline/2009/10/ WC500004883.pdf (accessed January 30, 2014).

18. Food and Drug Administration Safety and Innovation Act (FDASIA) Amendment to the Federal Food Drug and Cosmetic Act, Public law 112-114, July 9, 2012. http://www.gpo.gov/fdsys/pkg/PLAW-112publ144/pdf/ PLAW-112publ144.pdf (accessed January 30, 2014).

19. GUIDANCE FOR INDUSTRY Expedited Programs for Serious Conditions - Drugs and Biologics, Draft June 2013. http://www.fda.gov/downloads/Drugs/Gui danceComplianceRegulatoryInformation/Guidances/ UCM358301.pdf (accessed January 30, 2014).

20. COUNCIL RECOMMENDATION of 8 June 2009 on an action in the field of rare diseases. Official Journal of the European Union. 2009; 3.7.2009. http://eur-lex.europa. eu/LexUriServ/LexUriServ.do? uri=OJ:C:2009:151:0007 :0010:EN:PDF (accessed January 30, 2014).

21. Clinical Development of Nitisinone for Alkaptonuria. http://cordis.europa.eu/projects/rcn/106157_en.html (accessed January 30, 2014).

22. Hughes-Wilson W, Palma A, Schuurman A, Simoens S. Paying for the orphan drug system: Break or bend? Is it time for a new evaluation system for payers in Europe to take account of new rare disease treatments? Orphanet J Rare Dis. 2012; 7:74.

(Received January 30, 2014; Accepted February 6, 2014) 